\title{
Successful Treatment of Polycystic Ovarian Syndrome, Nonalcoholic Fatty Liver Disease and Infertility with Chinese Herbal Medicine: A Case Report Xianqin $\mathbf{Q u}^{*}$ and Madeleine Ong
}

School of Medical \& Molecular Biosciences, University of Technology Sydney, NSW 2007, Australia

\begin{abstract}
This case report details a 30 year old woman with hyperlipidemia, Nonalcoholic Fatty Liver Disease (NAFLD), Polycystic Ovarian Syndrome (PCOS) and infertility. Her impaired liver function has prevented the use of pharmaceutical drugs (statins and metformin). The patient therefore underwent two stages of Chinese herbal medicine treatment: the first formula to ameliorate hyperlipidemia and amenorrhea; and the second formula to improve ovarian dysfunction as well as glucose and lipid metabolism. Over 8 months of treatment, the patient achieved pregnancy and a natural birth without developing gestational diabetes. While the treatment of coexisting metabolic syndrome conditions is often complex and difficult, this case report highlights how Chinese herbal medicine may offer a successful treatment option for women with multiple metabolic abnormalities and associated infertility.
\end{abstract}

Keywords: Polycystic ovarian syndrome; Non-alcoholic fatty liver disease; Metabolic syndrome; Infertility; Chinese herbal medicine

\section{Introduction}

Polycystic Ovarian Syndrome (PCOS) is the most common reproductive endocrine disease with a prevalence of $5-10 \%$ in women of reproductive age [1]. The clinical features of PCOS are heterogeneous and vary in intensity. Hirsutism, oligomenorrhea or amenorrhea contributing to chronic anovulation and infertility are the most frequent conditions observed [2]. PCOS is also associated with several metabolic disturbances which characterize metabolic syndrome such as obesity, dyslipidemia and insulin resistance [3]. More recently, increased prevalence of Nonalcoholic Fatty Liver Disease (NAFLD) has been reported in patients with PCOS [4]. NAFLD entails a spectrum of fat accumulation and inflammation of the liver unrelated to alcohol consumption. NAFLD is primarily asymptomatic, but can be diagnosed by elevation of serum Alanine Amino Transferase (ALT) and Aspartate Transaminase (AST) with hepatic steatosis confirmed by ultrasonography and liver biopsy [5].

The combination of PCOS and NAFLD place patients at high risk for type 2 diabetes, visceral obesity and resistance to insulin as well as a manifold of other traditional cardiovascular risk factors especially intolerance, inflammation and procoagulation [6,7]. These in turn adversely affect pregnancy rates for women of reproductive age. Furthermore, pregnant women with a history of PCOS and NAFLD commonly develop gestational diabetes due to a predisposition to insulin resistance and have an increased risk for preeclampsia and preterm births [8-10].

Given the decreased fertility rate and adverse outcomes of pregnancy in women with NAFLD and PCOS, it is necessary to optimally manage these complex metabolic and reproductive disorders. There are several approaches to the treatment of subfertility found in women with PCOS. Lifestyle interventions are generally applied first, followed by oral treatments, and ending with more intensive strategies such as laparoscopic ovarian drilling and assisted reproductive techniques $[1,11]$. These methods have a limited effect, are costly or invasive. Moreover, some women with coexisting PCOS and metabolic syndrome may be unsuitable candidates for pharmaceutical medications due to impaired liver function. In this report we present a complex case of NAFLD, PCOS and infertility treated by Chinese herbal medicine.

\section{Case in Detail}

A 30 year-old woman visited our clinic for Chinese medicine treatment of oligomenorrhea with her cycles ranging between 40-60 days and an absence of menses for the last 4 months. Despite being married for four years, she had failed to fall pregnant with no history of use of the contraceptive pill or other forms of contraception. Her husband was of the same age and had no remarkable health problems. The patient reported a strong appetite leading to weight gain of more than $5 \mathrm{~kg}$ in the last two years and a Body Mass Index (BMI) of 29 $\mathrm{kg} / \mathrm{m}^{2}$. Physical examination revealed moderate hirsutism. Laboratory evaluations indicated an elevated Aspartate Transaminase (AST) level of $46 \mathrm{U} / \mathrm{L}$, Alanine Transaminase (ALT) level of $106 \mathrm{U} / \mathrm{L}$ and gammaGlutamyl Transferase (GGT) level of $64 \mathrm{U} / \mathrm{L}$ (normal ranges of AST, ALT and GGT all $<30$ ); fasting serum cholesterol level of $8.0 \mathrm{mmol} / \mathrm{L}$ (normal range 3.5-5.4) and triglycerides level of $2.9 \mathrm{mmol} / \mathrm{L}$ (normal range $0.1-2.0$ ). While there was a normal fasting plasma glucose level at $5.3 \mathrm{mmol} / \mathrm{L}$ (normal range 3.4-7.7) impaired glucose tolerance was revealed. Abdominal ultrasound showed an increased echodensity of the liver. This was consistent with moderate fatty infiltration, characteristic of simple steatosis in NAFLD. Pelvic ultrasound also revealed that both left and right ovaries contained multiple small follicles (each side 10 to 15 follicles measuring up to $6 \mathrm{~mm}$ ).

After an initial treatment with Chinese herbal medicine to induce menstruation, the patient gained her menses and subsequently underwent hormone testing on day 21 of her menstrual cycle. This identified Luteinising Hormone (LH) levels of 42 IU/L (normal range 2-15), Follicle-Stimulating Hormone (FSH) levels of $15 \mathrm{IU} / \mathrm{L}$ (normal range 2-12), estrogen levels of $3520 \mathrm{pmol} / \mathrm{L}$ (normal range 370-770) and progesterone levels of $3 \mathrm{nmol} / \mathrm{L}$ (normal range 10.6-89.1). The androgens were abnormally high with a testosterone level of $3.8 \mathrm{nmol} / \mathrm{L}$ (normal range $<2.6$ ) and free androgen index of 9.7\% (normal range $<7.2$ ) while De-hydro-epi-andro-sterone sulphate (DHEAS) had a level of $4.9 \mu \mathrm{mol} / \mathrm{l}$ (normal range 0.9-12) and Sex Hormone-Binding Protein (SHBP) levels of $39 \mathrm{nmol} / \mathrm{l}$ (normal range 20-118) within normal

*Corresponding author: Xianqin Qu, School of Medical \& Molecular Biosciences University of Technology Sydney, NSW 2007, Australia, Tel: +61-2-9514 7852; Fax: +61-2-9514 8206; E-mail: Xianqin.Qu@uts.edu.au

Received June 08, 2015; Accepted July 01, 2015; Published July 10, 2015

Citation: Qu X, Ong M (2015) Successful Treatment of Polycystic Ovarian Syndrome, Nonalcoholic Fatty Liver Disease and Infertility with Chinese Herbal Medicine: A Case Report. Endocrinol Metab Synd 4: 183. doi:10.4172/21611017.1000183

Copyright: (c) 2015 Qu X, et al.. This is an open-access article distributed under the terms of the Creative Commons Attribution License, which permits unrestricted use, distribution, and reproduction in any medium, provided the original author and source are credited. 
ranges. According to the Rotterdam criteria this patient was diagnosed with PCOS [2].

\section{Treatment}

The patient was prescribed Lipitor for her hyper-lipidemia and referred to see a gynecologist for infertility. However, she was worried about hepatic-related side effects of the medication due to her impaired liver function. The patient therefore sought out Chinese medicine treatment at our clinic to maintain a regular menstrual cycle, lose weight, reduce cholesterol, normalize her liver function and fall pregnant.

Chinese Herbal Medicines (CHM) have been used traditionally to treat both gynaecological and liver related disorders. A special feature of CHM is that it utilizes formulas containing several herbs to ameliorate a group of clinical and biochemical abnormalities. For this patient, a basic formula was prescribed including Radix Angelicae sinensis (dang gui) 6 g, Radix Rehmannia glutinosa (shu di huang) 10 g, Semen Cuscutae Chinensis (tu si zi)15 g, Radix Salvia miltiorrhizae (dan shen)15 g, Fructus Crataegus pinnatifida (shan zha)15 g, Fructus Gardenia florida (zhi zi) $6 \mathrm{~g}$ and Rhizoma Coptis chinensis (huang lian) $6 \mathrm{~g}$. This formula was prepared as an $800 \mathrm{~mL}$ raw herbal decoction which was to be consumed four times over two days $(200 \mathrm{~mL}$ twice per day). The aims of this particular herbal treatment were to regulate the menstrual cycle, promote ovulation, control metabolic abnormalities, reduce hepatic fat and reduce body weight. The patient therefore received 10 weeks of treatment.

\section{Follow Up and Result}

During the 10 weeks of treatment, the patient's menstrual cycle became regular with cycles shortening to 32-36 days. A blood test was also performed at this stage and revealed improvement of fasting serum lipids including cholesterol from 8.0 down to $5.8 \mathrm{mmol} / \mathrm{L}$ and triglycerides from 2.9 to $1.7 \mathrm{mmol} / \mathrm{L}$, as well as reduced serum hepatic enzymes AST 29, ALT 51 and GGT 33 U/L. After these 10 weeks, the treatment then continued with a new focus on promoting fertility to achieve pregnancy. The second formula included Radix Rehmannia glutinosa (shu di huang)15 g, Semen Cuscutae chinensis (tu si zi) $15 \mathrm{~g}$, Semen Morus multicaulis (sang shen zi) 15 g, Fructus Lycium barbarum (gou qi zi) 10 g, Herba Epimedium grandiflorum (yin yang huo) 10 g, Radix Salvia miltiorrhizae (dan shen)10 g, and Rhizoma Coptis chinensis (huang lian) $6 \mathrm{~g}$ and was prepared the same way as described previously. A day 3 hormone test performed 6 months after the initial hormone test revealed a remarkable change in testosterone (from 3.8 to $1.3 \mathrm{nmol} / \mathrm{L}$ ) and free androgen index (from $9.7 \%$ to $4.8 \%$ ) with female hormones all within normal reference ranges for the follicular stage. Pregnancy was also achieved after 8 months of treatment with Chinese herbal medicines. The herbal treatment was then ceased after confirmation of pregnancy. During her pregnancy she did not develop gestational diabetes and had a natural labour giving birth to a healthy baby boy.

\section{Discussion}

This report details a case where a woman of childbearing age had profound hyperlipidemia, fatty liver disease and PCOS contributing to anovulatory infertility. For her complex disorders, a monotherapy approach may not have been effective. On the other hand, a combination of medications to treat PCOS and NAFLD simultaneously can be contradictory. For example, impaired liver function in this case hinders the use of lipid-lowering agents such as statins due to controversy of these drugs leading to liver toxicity [12]. The patient had previously complied with lifestyle modifications, however had unsatisfactory results due to the complexity and severity of her metabolic abnormalities. We achieved her pregnancy and a healthy live birth with two sets of Chinese herbal formulae containing herbs with anti-obesity, lipid lowering and hepatoprotective effects to benefit both liver and ovary function.

In the treatment of PCOS, the contraceptive pill is often administered to suppress androgens and regulate the menstrual cycle [13]. However, this treatment strategy was not suitable for this patient who wanted to get pregnant as the OCT inhibits ovulation and prevents sperm penetration. Additionally the contraceptive pill does not treat NAFLD and is associated with the side effect of weight gain which can further aggravate PCOS and NAFLD related symptoms. Management of PCOS with pharmaceutical medications of metformin and clomiphene citrate has been well documented [11,13]. Clomiphene is used to increase ovarian stimulation to induce ovulation. However, clomiphene has limited efficacy and is associated with various side effects as well as hyper-ovarian stimulation [14]. Metformin is an insulin sensitiser that may improve insulin resistance to ameliorate both PCOS and NAFLD. However, metformin is not recommended for patients with liver dysfunction (elevated serum AST and/or ALT) [15]. Additionally, the patient presented with an absence of menses for four months and metformin may have a limited effect on inducing menstruation. For these reasons a natural approach was adopted.

In regards to Chinese herbal medicine there is considerable knowledge concerning both efficacy and safety for gynaecological disorders, such as irregular menstruation, amenorrhea and PCOS with infertility $[16,17]$. An advantage of $\mathrm{CHM}$ treatment is that it utilizes individualized formulas tailored to a patient's condition. In this case, the first formula was used to improve her 'blood stasis syndrome' which in TCM is a phenomena related to amenorrhea, to induce her menses. The second formula mainly addressed the ovarian dysfunction with some of the herbal ingredients, such as Radix Salvia miltiorrhizae, and Rhizoma Coptis chinensis, also having beneficial effects on metabolic syndrome [18]. As a result, the treatment achieved not only her conception but also prevention of gestational diabetes during the pregnancy in this woman with multiple metabolic and reproductive conditions. CHM as an effective treatment for PCOS with subfertility has been evidenced by previous clinical studies [17]. These studies included 344 subjects who were diagnosed with PCOS and subfertility without NAFLD and hyperlipidaemia. The result of our case report demonstrates that CHM may be effective for complex metabolic and reproductive disorders with infertility.

\section{Conclusion}

In summary, herbal medicinal formulas may work on multiple targets simultaneously to improve metabolic syndrome manifestations such as PCOS, especially when there is more than one metabolic condition involved. More specifically, this case report demonstrates how herbal medicine can be applied to encourage the regularity of the menstrual cycle, promote ovulation and improve lipid metabolism to ameliorate PCOS and NAFLD related symptoms whilst also facilitating conception. The successful treatment with CHM for this case highlights a potential of natural therapy to manage coexisting NAFLD, PCOS and subfertility cohesively. The reliability of CHM for the treatment of complex endocrinal and reproductive disease is therefore worthwhile and needs to be implemented though rigorously designed randomized and controlled clinical trials. 
Citation: Qu X, Ong M (2015) Successful Treatment of Polycystic Ovarian Syndrome, Nonalcoholic Fatty Liver Disease and Infertility with Chinese Herbal Medicine: A Case Report. Endocrinol Metab Synd 4: 183. doi:10.4172/2161-1017.1000183

\section{References}

1. Lauritsen MP, Bentzen JG, Pinborg A, Loft A, Forman JL, et al. (2014) The prevalence of polycystic ovary syndrome in a normal population according to the Rotterdam criteria versus revised criteria including anti-Müllerian hormone. Hum Reprod 29: 791-801.

2. Azziz R, Carmina E, Dewailly D, Diamanti-Kandarakis E, Escobar-Morreale $H$, et al. (2006) Criteria for defining polycystic ovary syndrome as a predominantly hyperandrogenic syndrome: an Androgen Excess Society guideline. J Clin Endocrinol Metab 91: 4237-4242.

3. Baranova A, Tran TP, Biererdinc A, Younossi M (2011) Systematic review: association of polycystic ovary syndrome with metabolic syndrome and nonalcoholic fatty liver disease. Aliment Pharmacol Ther 33: 801-814.

4. Kelley CE, Brown AJ, Diehl A.M, Setji TL (2014) Review of nonalcoholic fatty liver disease in women with polycystic ovary syndrome. World J Gastroenterol 20: 14172-14184.

5. Almeda-Valdés P, Cuevas-Ramos D, Aguilar-Salinas C (2009) Metabolic syndrome and non-alcoholic fatty liver disease. Ann Hepatol, 8: S18-S24.

6. Vassilatou E (2014) Nonalcoholic fatty liver disease and polycystic ovary syndrome. World J Gastroenterol 20: 8351-8363.

7. Manneras-Holm L, Baghaei F, Holm G, Janson PO, Ohlsson C, et al. (2011) Coagulation and fibrinolytic disturbances in women with polycystic ovary syndrome. Clin Endocrinol Metab 96: 1068-10676.

8. Rojas J, Chávez-Castillo M, Bermúdez V (2014) The role of metformin in metabolic disturbances during pregnancy: Polycystic ovary syndrome and gestational diabetes mellitus. Am J Obstet Gynecol 2014: 797681.

9. Hagström H, Höijer J, Ludvigsson JF, Bottai M, Ekbom A, et al. (2015) Adverse outcomes of pregnancy in women with non-alcoholic fatty liver disease. Liver Int 2015 doi: 10.1111/liv.12902. [Epub ahead of print]

10. De Frène V, Vansteelandt S, T'Sjoen G, Gerris J, Somers S, et al. (2014) A retrospective study of the pregnancy, delivery and neonatal outcome in overweight versus normal weight women with polycystic ovary syndrome. Hum Reprod 2014 29: 2333-8.

11. Panidis D, Tziomalos K, Papadakis E, Katsikis I (2013) Infertility Treatment in Polycystic Ovary Syndrome: Lifestyle Interventions, Medications and Surgery Front Horm Res 40: 128-141.

12. Kinnman N, Hultcrantz R (2001) Lipid lowering medication and hepatotoxicity. Intern Med 250: 183-185.

13. Sirmans SM, Pate KA (2014) Epidemiology, diagnosis, and management of polycystic ovary syndrome. Clin Epidemiol 6: 1-13.

14. Legro RS, Brzyski RG, Diamond MP, Coutifaris C, Schlaff WD, et al. (2014) Letrozole versus Clomiphene for Infertility in the Polycystic Ovary Syndrome. N Engl J Med 371: 119-129.

15. Dalpiaz A, Fonseca V, Dalpiaz A, Tan MH (2007) Spectrum of liver disease in type 2 diabetes and management of patients with diabetes and liver disease. Diabetes Care 30: 734-743.

16. Tan L, Tong Y, Sze S.C.W, Xu M, Shi Y, et al. (2012) Chinese Herbal Medicine for Infertility with Anovulation: A Systematic Review. J Altern Complement. Med 18: $1087-1100$

17. Zhang J, Li T, Zhou L, Tang L, Xu L, et al. (2010) Chinese herbal medicine for subfertile women with polycystic ovarian syndrome. Cochrane Database Syst Rev 8: 1-45

18. Tan Y, Kamal M.A, Wang ZZ, Seale JP, Qu X et al. (2011) Chinese herbal extracts (SK0506) as a potential candidate for the therapy of metabolic syndrome. Clin Sci 120: 297-305.
Citation: Qu X, Ong M (2015) Successful Treatment of Polycystic Ovarian Syndrome, Nonalcoholic Fatty Liver Disease and Infertility with Chinese Herbal Medicine: A Case Report. Endocrinol Metab Synd 4: 183. doi:10.4172/21611017.1000183
Submit your next manuscript and get advantages of OMICS Group submissions

Unique features:

User friendly/feasible website-translation of your paper to 50 world's leading languages

Audio Version of published paper

Digital articles to share and explore

Special features:

350 Open Access Journals

30,000 editorial team

21 days rapid review process

Quality and quick editorial, review and publication processing

Indexing at PubMed (partial), Scopus, EBSCO, Index Copernicus and Google Scholar etc

Sharing Option: Social Networking Enabled

Authors, Reviewers and Editors rewarded with online Scientific Credits

Better discount for your subsequent ar

Submit your manuscript at: http://www.editorialmanager.com/biochen 